\title{
The formation of classical defects after a slow quantum phase transition
}

\author{
R.J. Rivers ${ }^{\mathrm{a}, 1}$, F.C. Lombardo ${ }^{\mathrm{b}}$, F.D. Mazzitelli ${ }^{\mathrm{b}}$ \\ ${ }^{a}$ Centre of Theoretical Physics, University of Sussex, Brighton BN1 9QJ, United Kingdom \\ b Departamento de Física, Facultad de Ciencias Exactas y Naturales, Universidad de Buenos Aires, Ciudad Universitaria, \\ Pabellón I, 1428 Buenos Aires, Argentina
}

Received 3 May 2002; accepted 30 May 2002

Editor: P.V. Landshoff

\begin{abstract}
Classical defects (monopoles, vortices, etc.) are a characteristic consequence of many phase transitions of quantum fields. We show a model in which the onset of classical probability distributions, for the long-wavelength modes at early times, allows the identification of line-zeroes of the field with vortex separation. We obtain a refined version of Kibble's causal results for defect separation, but from a completely different approach. It is apparent that vortices are not created from thermal fluctuations in the Ginzburg regime. ๑ 2002 Elsevier Science B.V. All rights reserved.
\end{abstract}

Because phase transitions take place in a finite time, causality requires correlation lengths to remain finite. As a result, scalar order parameter fields $\phi(\mathbf{x})$ become frustrated, and topological defects arise so as to reconcile field phases between different correlation volumes [1,2]. For example, the breaking of the GUT symmetry in the early universe gives rise to monopoles, the most familiar defect. However, they are but one of many possibilities, that include cosmic strings (vortices), which may be the source of high energy cosmic rays, as well as contributing to structure formation in the early universe.

For the weak-coupling theories of the early universe, defects have non-perturbatively large masses,

E-mail addresses: r.rivers@ic.ac.uk (R.J. Rivers), lombardo@df.uba.ar (F.C. Lombardo),fmazzi@df.uba.ar (F.D. Mazzitelli).

${ }^{1}$ Permanent address: Imperial College, London SW7 2BZ. comparable to the temperature scale $k_{\mathrm{B}} T_{\mathrm{c}}$ at which the transition takes place. Thus monopoles produced in the early universe at a GUT transition might be expected to have a mass $m \sim 10^{16} \mathrm{GeV}$. For this reason alone, they and other defects are manifestly classical, even though the phase transition that produced them is intrinsically quantum mechanical. In this Letter we show how the creation of classical defects comes about for simple temperature quenches with a finite quench rate through the critical temperature $T_{\mathrm{c}}$. (The case of an instantaneous quench has been considered elsewhere [3].) Finite quench rates have been studied by Kibble [4], using causality bounds, from which he has derived simple scaling laws that incorporate meanfield dimensional analysis.

Although the equilibrium correlation length $\xi_{\text {eq }}(t)$ diverges at the transition, the true correlation length $\xi(t)$ does not. Thus, causality imposes a maximum rate at which the correlation length can grow and 
hence a maximum correlation length, $\bar{\xi}$, at the onset of the transition. At the same time, causality imposes a horizon outside which the fields are uncorrelated. If we simply relate the correlation length $\bar{\xi}$ and the separation length between vortices $\bar{\xi}_{\text {def }}$ by $\bar{\xi}=$ $O\left(\bar{\xi}_{\text {def }}\right)$, then the density of defects is bounded, and calculable, at their moment of formation.

If, in the vicinity of $T_{\mathrm{c}}, d T / d t=-T_{\mathrm{c}} / \tau_{\mathrm{Q}}$, then dimensional analysis suggests that the earliest possible time at which defects could be formed after the onset of the transition is $t=O\left(t_{\mathrm{K}}\right)$, where $t_{\mathrm{K}}=$ $\left(\tau_{\mathrm{Q}} / \mu^{2}\right)^{1 / 3}$. This is deduced from $\dot{\xi}_{\text {eq }}\left(t=t_{\mathrm{K}}\right)=$ -1 , where $\xi_{\text {eq }}(t)=\left|m^{-1}(t)\right|=\mu^{-1} \sqrt{\tau_{\mathrm{Q}} / t}$ ( $\mu$ is the particle mass). We shall use this as a benchmark in our calculations. Therefore, from causality arguments, we derive the bound [1]

$\bar{\xi}_{\mathrm{K}}=\bar{\xi}\left(t=t_{\mathrm{K}}\right)=\bar{\xi}_{\mathrm{eq}} \approx \mu^{-1}\left(\tau_{\mathrm{Q}} \mu\right)^{1 / 3}$,

leading to the estimate $\bar{n}_{\text {def }}=O\left(\bar{\xi}_{\mathrm{K}}^{-2}\right)$ for the defect density at the time of their production.

But there is an alternative scenario for counting defects after the transition is completed. Simple defects have false ground-state or vacuum at their cores where the field vanishes. Under suitable conditions, the separation length for defects is more sensibly derived by counting zeroes of the field as $\bar{\xi}_{\text {def }}=O\left(\bar{\xi}_{\text {zero }}\right)$, where $\bar{\xi}_{\text {zero }}$ measures the separation of field zeroes. However, in principle, $\bar{\xi}_{\text {zero }}$ and $\bar{\xi}_{\text {def }}$ are different correlation lengths, since not all zeroes are candidate defects (because zeroes occur on all scales). One needs to count zeroes of an appropriately coarse-grained field, in which structure on a scale smaller than an specific classical vortex size $\xi_{0} \sim \mu^{-1}=\Lambda$, is not present. At a given time $t^{*}$ the separation of zeroes is $\bar{\xi}_{\text {zero }}^{\Lambda}\left(t^{*}\right)$. Then, in order that line-zeroes can be identified with classical defects vortex cores, and $\bar{\xi}_{\text {zero }}^{\Lambda}\left(t^{*}\right)$ with $\bar{\xi}_{\text {def }}$ we need satisfy, essentially, two important conditions [5]: (I) the separation between zeroes must be insensitive to the cutoff scale $\Lambda^{-1} \sim \mu^{-1}$; and (II) the energy in field gradients should be commensurate with the energy in classical defects with the same density as that of line-zeroes.

We will show how decoherence can help to satisfy these two conditions. For weak couplings (massive defects) our main prediction agrees qualitatively with the causal (dimensional) prediction for counting defects, but here from a completely different point of view, in which classicalisation of long-wavelength (unstable) modes of the field warrants the identification of linezeroes with defects at the time in which the transition has been completed.

There are further complications according as the symmetries are global or gauged [6], but the simplest of all are global, and it is these that we shall consider here. We have global vortices in mind, the simplest sensible defects, in which case the order parameter field $\Phi(\mathbf{x})$ is a complex scalar $\Phi=\left(\phi_{1}+i \phi_{2}\right) / \sqrt{2}$, with action

$S[\Phi]=\int d^{4} x\left\{\frac{1}{2} \partial_{\mu} \phi_{a} \partial^{\mu} \phi_{a}+\frac{1}{2} \mu^{2} \phi_{a}^{2}-\frac{\lambda}{4}\left(\phi_{a}^{2}\right)^{2}\right\}$.

With $\mu^{2}>0$, the $O(2)$ symmetry is broken at the scale $|\Phi|=\eta, \eta^{2}=\mu^{2} / 2 \lambda$. (Generalisation to global monopoles, or even domain walls, is straightforward.)

Global vortices are line defects in the field, solutions to

$\frac{\delta S[\Phi]}{\delta \Phi}=0$,

around which the field phase $\theta\left(\Phi=h e^{i \theta}\right)$ changes by $2 \pi$. Considered as tubes of 'false' vacuum, with cold thickness $O\left(\mu^{-1}\right)$, they have a line of field zeroes at their cores and, once the transition is complete, energy per unit length $\sigma=O\left(\mu^{2} / \lambda\right)$ (up to multiplicative logarithmic terms $\left.\ln \left(\mu \xi_{\text {def }}\right)\right)$.

There is no unambiguous definition of the onset of classical behaviour, least of all for the production of classical defects. Our approach stresses the role of classical stochastic field equations. They arise in two forms, in Lagrange's form as stationary phase approximations to the evolution kernel of the density matrix, or in Hamilton's form as the dominant path in phase space of the evolution kernel of the Wigner functional. Initially we shall concentrate on the former. In general, if the system is closed, quantum interference between different field configurations forbids us from identifying the dynamical solutions to (3) for the evolving system as describing real defects. However, if the system is open, the environment with which it interacts can eliminate the interference, in which case the system can be said to have decohered.

In practice, the systems of interest to us are open. This is true, firstly, in the trivial sense that, in the absence of superselection rules, our order parameter fields $\Phi(\mathbf{x})$ interact with everything else in the uni- 
verse (termed $\chi(\mathbf{x})$ ), but this environment is traced over when looking at the transition. Further, for the continuous transitions of (2) that will interest us here, field ordering takes place through the exponential growth of the unstable $(k<\mu)$ long-wavelength modes $\Phi_{<}(\mathbf{x})$ of $\Phi(\mathbf{x})$. The stable $(k>\mu)$ shortwavelength modes $\Phi_{>}(\mathbf{x})$ do not become classical and behave as a further part of the environment in which the long-wavelength modes, which constitute our 'system', decohere. As a consequence, the interiors of vortices are not classical. Since it is the field profile in the interior of the classical vortex that carries the nonperturbatively large energy or tension, this might be thought to be damaging, but we will see that it is not the case. In particular, topological charge can be classical. As a result, non-classical cores are irrelevant as far as counting defects is concerned.

Our classical equations are, most simply, stationary phase approximations to the evolution of the reduced density matrix $\rho_{\mathrm{r}}\left[\phi_{<}^{+}, \phi_{<}^{-}, t\right]=\left\langle\phi_{<}^{+}\left|\hat{\rho}_{\mathrm{r}}(t)\right| \phi_{<}^{-}\right\rangle$, obtained by tracing out the environments $\chi$ and $\phi_{>}$ (we are using $\phi_{>}$to denote the real Cartesian doublet). In a field basis (driven by our need to describe defects through field equations) $\rho_{\mathrm{r}}\left[\phi_{<}^{+}, \phi_{<}^{-}, t\right]$ evolves in time by means of the coarse-grained effective action (CGEA) $A\left[\phi_{<}^{+}, \phi_{<}^{-}\right]$, as we have shown in Refs. [7,8] (and in a more detailed version in [9]). The CGEA takes into account the effect of the environment on the dynamics of the system. We regard the imaginary part of $A$ as coming from a classical noise source $\zeta(x)$ (or several such sources), say. If its probability distribution is $p[\zeta]$, then the CGEA can be rewritten as $[8,10]$

$$
\begin{aligned}
A & {\left[\phi_{<}^{+}, \phi_{<}^{-}\right] } \\
& =-\frac{1}{i} \ln \int \mathcal{D} \zeta p[\zeta] \exp \left\{i S_{\mathrm{eff}}\left[\phi_{<}^{+}, \phi_{<}^{-}, \zeta\right]\right\},
\end{aligned}
$$

where

$$
\begin{aligned}
& S_{\text {eff }}\left[\phi_{<}^{+}, \phi_{<}^{-}, \zeta\right] \\
& \quad=\operatorname{Re} A\left[\phi_{<}^{+}, \phi_{<}^{-}\right]-\int d^{4} x[\Delta(x) \zeta(x)],
\end{aligned}
$$

and $\Delta(x)$ depends on the way $[8,11,12]$ in which the environment couples to the $\phi_{<}$field. For the simplest case of biquadratic couplings (e.g., $\phi_{<}^{2} \chi^{2}$ or the inevitable $\left.\phi_{<}^{2} \phi_{>}^{2}\right)$, we find $\Delta=\frac{1}{2}\left(\left|\Phi_{<}^{+}\right|^{2}-\left|\Phi_{<}^{-}\right|^{2}\right)$.
The stationary phase equation, obtained by taking the functional variation

$$
\begin{aligned}
& \left.\frac{\delta S_{\mathrm{eff}}\left[\phi_{<}^{+}, \phi_{<}^{-}, \zeta\right]}{\delta \phi_{a<}^{+}}\right|_{\phi_{<}^{+}=\phi_{<}^{-}} \\
& \quad=\left.\frac{\delta \operatorname{Re} A\left[\phi_{<}^{+}, \phi_{<}^{-}, \zeta\right]}{\delta \phi_{a<}^{+}}\right|_{\phi_{<}^{+}=\phi_{<}^{-}}-\left.\zeta \frac{\delta \Delta}{\delta \phi_{a<}^{+}}\right|_{\phi_{<}^{+}=\phi_{<}^{-}} \\
& \quad=0,
\end{aligned}
$$

is a generalised Langevin equation, with classical noise $\zeta$ and dissipation, to satisfy the fluctuationdissipation theorem (see Ref. [9] for a complete deduction of Eq. (6)). If we look for vortex solutions $\Phi_{<}^{\zeta}$ to (6), modelled on the vortex solutions to (3), we shall find ourselves in some difficulty at early times, in that the noise will cause fluctuations (e.g., small vortex loops) that will make the identification of a single vortex difficult.

However, the existence of classical defects depends on more than clean vortex solutions to Eq. (6). The absence of quantum interference between the stationary phase solutions $\phi_{<}^{\zeta}$ to the classical stochastic equations and their neighbours is manifest through the increasing diagonalisation of $\rho_{\mathrm{r}}\left[\phi_{<}^{+}, \phi_{<}^{-}, t\right]$. This leads to the crucial notion of a decoherence time $t_{\mathrm{D}}$, after which $\rho_{\mathrm{r}}\left[\phi_{<}^{+}, \phi_{<}^{-}, t\right]$ (or, more exactly, its real part) is effectively diagonal. Eq. (6) is the classical stochastic equation that we are looking for but, as it stands, is only guaranteed to describe classical defects after decoherence.

A digression is necessary. There is not a universal decoherence time $t_{\mathrm{D}} \cdot t_{\mathrm{D}}(k)$ depends on wavelength. Long-wavelength field modes decohere first, shorter ones later, and those with $k>\mu$ never. To make the problem of diagonalisation tractable we have restricted ourselves to field configurations $\Phi_{<}^{ \pm}\left(k_{0}\right)$ peaked around a particular wavenumber $k_{0}$ [3,7-9]. The order parameter itself corresponds to $k_{0}=0$ but, more generally, we are motivated by the way in which the power in the field fluctuations is increasingly peaked around long-wavelengths in the early stage of the transition $[5,13,14]$. That is, if the power spectrum $P(k, t)$ of the field fluctuations is defined by

$$
\begin{aligned}
G_{<}(r, t) & =\left\langle\Phi_{<}(\mathbf{x}) \Phi_{<}^{*}(\mathbf{0})\right\rangle_{t} \\
& =\int_{k<\mu} \frac{d^{3} k}{(2 \pi)^{3}} P(k, t) e^{i \mathbf{k} . \mathbf{x}}
\end{aligned}
$$


then, provided the quench is fast enough, $k^{2} P(k, t)$ rapidly develops a 'Bragg' peak at $k^{2}=k_{\mathrm{p}}^{2}(t)<$ $\mu^{2}$. A sufficient condition for this to happen is that $\mu \tau_{Q} \lesssim \eta / \mu$ [14]. Tighter, but less transparent bounds can be given [14]. We restrict ourselves to quenches permitting such a dominant momentum peak and identify $k_{0}$ with it. The relevance of this is that it is the peak in $k^{2} P(k, t)$ that sets the scale for the separation of line-zeroes in the field $\phi$. Once $k_{\mathrm{p}}^{2}(t) \ll \mu^{2}$ it does not matter where the boundary $\Lambda=O(\mu)$ between system and environment is set. Since defects can be characterised by the line-zeroes at their cores [15], it is the dominant wavenumber $k_{0}$ that, after the onset of classical behaviour, sets the density of the vortices that we wish to determine. This peak at $k_{0}$ also warrants the fulfillment of condition (I).

This peaking stops once the field has sampled the ground state values at $|\Phi|=\eta$. It does this by the spinodal time $t_{\mathrm{sp}}$, defined as that value of $t$ (after the critical temperature $T_{\mathrm{c}}$ has been crossed) for which $\left\langle\left|\Phi_{<}\right|^{2}\right\rangle_{t}=\eta^{2}$. For the critical temperature $T_{\mathrm{c}}$ traversed at a finite rate $\tau_{\mathrm{Q}}^{-1}\left(\mu \tau_{\mathrm{Q}} \gg 1\right)$ we find $[5,14,16]$

$\exp \left\{\frac{4}{3}\left(\frac{t_{\mathrm{sp}}}{t_{\mathrm{K}}}\right)^{3 / 2}\right\} \sim \frac{\mu \eta^{2} t_{\mathrm{K}}^{2}}{T_{\mathrm{c}}}$.

In Eq. (8) the time $t_{\mathrm{K}}$ is the same Kibble causal time introduced earlier (before Eq. (1)). Because of the exponential nature of the relationships, in practice $t_{\mathrm{sp}}=O\left(t_{\mathrm{K}}\right)$, but larger than it.

For $t \lesssim t_{\mathrm{sp}}$, perturbation theory can be used to calculate $t_{\mathrm{D}}$. Decoherence at scale $k_{0}$ occurs when the non-diagonal elements of the reduced density matrix are much smaller than the diagonal ones. In [9] we have evaluated $t_{\mathrm{D}}$ for the longest-wavelength modes, and found that $t_{\mathrm{D}}\left(k_{0}=0\right)<t_{\mathrm{sp}}$ for all sufficiently fast $\tau_{\mathrm{Q}}$ for a realistic class of environments (for the instantaneous quench see Refs. [7,8]). We have considered an explicit environment consisting of a large number $N$ of weakly coupled $\chi$ fields, with coupling constants $g_{i}$ satisfying $\sum_{i} g_{i}^{2}=O\left(\lambda^{2}\right)$, which permit a consistent loop expansion, with $\mu \ll T_{\mathrm{c}} \ll \eta$, and we continue with this hierarchy here. The expressions are complicated, even in one-loop, and the reader is referred there for details. The main result for the deco- herence time in a slow quench (and $k_{0} \approx 0$ ) is

$\exp \left\{\frac{4}{3}\left(\frac{t_{\mathrm{D}}}{t_{\mathrm{K}}}\right)^{3 / 2}\right\} \sim \frac{\eta^{2}}{T_{\mathrm{c}}^{2}} t_{\mathrm{K}} \sqrt{\mu T_{\mathrm{c}}}$.

Nevertheless, what interests us here is the shortestwavelengths that will have decohered by time $t_{\mathrm{sp}}$. Provided $k_{0}$ is not too close to $\mu$ it is sufficient to replace $\mu t_{\mathrm{D}}$ by $t_{\mathrm{D}}\left(k_{0}\right) \sqrt{\mu^{2}-k_{0}^{2}}$ in the calculations above to determine the bound on the decoherence time for the mode $k_{0}$. An immediate question is to ask what is the maximum value $k_{\max }$ for which $t_{\mathrm{D}}\left(k_{\max }\right)=t_{\mathrm{sp}}$. After this replacement in Eqs. (9) and (8) we obtain

$\frac{k_{\max }^{2}}{\mu^{2}} \approx \ln \left(\frac{T_{\mathrm{c}} \tau_{\mathrm{Q}}^{2 / 3}}{\mu^{1 / 3}}\right)$.

Alternatively, there is a time $t\left(k_{p}\right)$ when the mode $k_{p}$ would become the dominant wavenumber. This 'quantum time' is determined by the instabilities of the long-wavelength modes, which increasingly bunch about the wavenumber $k_{\mathrm{p}}^{2}(t) \sim \mu / t\left(k_{\mathrm{p}}\right)$ [14]. At time $t_{\mathrm{D}}\left(k_{\max }\right)=t_{\mathrm{sp}}$

$\frac{k_{p}^{2}\left(t_{\mathrm{sp}}\right)}{\mu^{2}} \sim \frac{1}{\mu t_{\mathrm{D}}} \sim \frac{1}{\mu t_{\mathrm{K}}} \ln \left(\frac{T_{\mathrm{c}}}{\mu \eta^{2} t_{\mathrm{K}}^{2}}\right)$.

Thus, the dominant modes at time $t_{\mathrm{sp}}$ have already decohered by this time.

Therefore, from the two previous equations we can see that

$\frac{k_{\max }^{2}}{k_{\mathrm{p}}^{2}} \approx 2 \mu t_{\mathrm{K}} \ln \left(\frac{T_{\mathrm{c}} \tau_{\mathrm{Q}}^{2 / 3}}{\mu^{1 / 3}}\right)>1$.

That is, the modes that determine defect separation are well decohered by time $t_{\mathrm{sp}}$. However, $t_{\mathrm{D}}(k) \rightarrow \infty$ as $k \rightarrow \mu^{-}$and the modes that characterise the classical profiles of individual defects $(k \gtrsim \mu)$ are not decohered.

This makes constructing exemplary solutions to Eq. (6), and averaging them, a suspect activity. Rather, we examine the second strand of classicality, that of classical probabilities, as expressed through classical correlations. This is understood as the quantum system, in full (field) phase-space, mimicking the classical dynamics. The comparison is made through the reduced Wigner functional, defined as

$$
\begin{aligned}
W\left[\phi_{<}, \pi_{<} ; t\right]=\int & \mathcal{D} \eta_{<} e^{i \int \pi_{<} \eta_{<}} \\
& \times\left\langle\phi_{<}-\eta_{<}\left|\rho_{\mathrm{r}}(t)\right| \phi_{<}+\eta_{<}\right\rangle .
\end{aligned}
$$


As is well known, $W\left[\phi_{<}, \pi_{<} ; t\right]$ itself is not guaranteed to be positive until decoherence has occurred [17], although

$$
\begin{aligned}
P\left[\phi_{<}\right]_{t} & =\int \mathcal{D} \pi_{<} W\left[\phi_{<}, \pi_{<} ; t\right] \\
& =\left\langle\phi_{<}\left|\rho_{\mathrm{r}}(t)\right| \phi_{<}\right\rangle>0,
\end{aligned}
$$

is always a true probability distribution for the field configuration $\phi_{<}$at time $t$.

After decoherence, when $W\left[\phi_{<}, \pi_{<} ; t\right]$ is positive, at least for long-wavelength modes, it can be identified with the Fokker-Planck probability distribution function $P_{t}^{\mathrm{FP}}\left[\phi_{<}, \pi_{<}\right]$, and $P\left[\phi_{<}\right]_{t}$ can be equally identified as the Fokker-Planck probability $P^{\mathrm{FP}}\left[\phi_{<}\right]_{t}$. Since the Fokker-Planck equation is the obverse of the Langevin equation, at this time we can equally average over Langevin noise $\zeta$ or over $P^{\mathrm{FP}}\left[\phi_{<}\right]_{t}$.

However, as we observed earlier, the validity of the classical stochastic equations is, of itself, not sufficient to guarantee classical defects with energy profiles derived from (3). We do not have defects until the fluctuations around them are negligible, and this is associated with the peaking of the power spectrum (and the validity of our approximation of adopting a dominant wavenumber) rather than decoherence or classical correlations directly $[5,18]$. To see this, suppose we can calculate $P\left[\phi_{<}\right]_{t}$ for all $t$. This permits us to calculate the equal time $n$-point correlation functions, in which we restore the subscripts $a, b, \ldots=1,2$,

$$
\begin{aligned}
& G_{<a b \ldots c}^{(\mathrm{n})}\left(\mathbf{x}_{1}, \ldots, \mathbf{x}_{n}, t\right) \\
& \quad=\int \mathcal{D} \phi_{<} P_{t}\left[\phi_{<}\right] \phi_{<a}\left(\mathbf{x}_{1}\right) \cdots \phi_{<c}\left(\mathbf{x}_{n}\right)
\end{aligned}
$$

for all times $t<t_{\mathrm{sp}}$. As we know, equal-time correlators are all we need to calculate densities of field zeroes (line-zeroes, etc.). However, if all we are going to use is $P\left[\phi_{<}\right]_{t}$, the diagonal matrix element of $\rho_{r}(t)$, there is no real need to construct the Wigner functional. We can just do a calculation of $P\left[\phi_{<}\right]_{t}$ from the start, along the lines of Ref. [13]. The weak interaction with the environment that is so successful in diagonalising the density matrix has only a small effect on the diagonal matrix elements $P\left[\phi_{<}\right]_{t}$ for most of the time until $t_{\mathrm{sp}}$.

The major characteristic of defects is their topological charge. We now see how localised topological charge precedes the appearance of vortices, and gives an estimate of their density when they do appear. Let us now consider the line-zero ensemble density $n_{\text {zero }}(t)$ and the line-zero separation $\xi_{\text {zero }}(t)$ for the long-wavelength mode fields. When the Gaussian approximation [19,20] is satisfied (as happens for $\left.t<t_{\mathrm{sp}}\right)$ they are determined completely by the shortdistance behaviour of $G_{<}(r, t)$ as

$n_{\text {zero }}(t)=\frac{1}{2 \pi \xi_{\text {zero }}^{2}(t)}=\frac{-1}{2 \pi} \frac{G_{<}^{\prime \prime}(0, t)}{G_{<}(0, t)}$.

Since $G_{<}(r, t)$ has short-wavelength modes on the scale of a classical vortex removed, $G_{<}(0, t)$ is finite. Initially, with $P(k, t)$ large for $k \gtrsim \mu, G_{<}(r, t)$ is very dependent on the value of the cut-off $\Lambda$. As a result, line-zeroes are extremely fractal, with a separation $\xi_{\text {zero }}(t)$ proportional to the scale at which they are viewed, and are certainly not candidates for defects. Only once the peak at $k=k_{0}$ is firmly in the interval $k<\mu$ does $\xi_{\text {zero }}(t)$ becomes insensitive to a cut-off $O\left(\mu^{-1}\right)$. This means that line-zeroes are straight at this scale, although they can be approximately random walks at much larger scales (condition (I)).

Further, for this early period, when the self-consistent mean-field approximation is valid, the field energy $\langle E\rangle_{t}$ of the system field $\phi_{<}$in a box of volume $\mathcal{V}$ becomes $[5,18]$

$$
\begin{aligned}
\langle E\rangle_{t}= & \mathcal{V}\left[\left\langle\left|\nabla \phi_{<}\right|^{2}\right\rangle_{t}+\lambda\left(\eta^{2}-\left\langle\left|\phi_{<}\right|^{2}\right\rangle_{t}\right)^{2}\right] \\
=\mathcal{V}\left[2 \pi n_{\text {zero }}(t) G_{<}(0, t)\right. & \\
& \left.+\lambda\left(\eta^{2}-G_{<}(0, t)\right)^{2}\right] \\
= & 2 \pi L_{\text {zero }}(t) G_{<}(0, t) \\
& +\mathcal{V}\left[\lambda\left(\eta^{2}-G_{<}(0, t)\right)^{2}\right] .
\end{aligned}
$$

As before, $\chi$-field fluctuations are absorbed in the definition of $\mu^{2}=\lambda \eta^{2}$. Eq. (16) is obtained by using (15) and $L_{\text {zero }}(t)=\mathcal{V} n_{\text {zero }}$ is the total length of line-zeroes, on a scale $\mu^{-1}$, in the box of volume $\mathcal{V}$.

We understand Eq. (17), valid from time $t=0$, when $G(0,0)=O\left(\mu^{2}\right)$, until time $t \approx t_{\mathrm{sp}}$, when $G\left(0, t_{\mathrm{sp}}\right) \simeq \eta^{2}$, as follows. At early times most of the system field energy (proportional to $\mathcal{V}$ ) is in fluctuations not associated with line zeroes, arising from the field potential. As time passes their energy density decreases as the system field approaches its posttransition value, becoming approximately zero. In addition there is a term, arising from the field gradients, 
proportional to the length $L_{\text {zero }}$ of line-zeroes, whose energy per unit length increases from $O\left(\mu^{2}\right)$ to $\eta^{2}$. At time $t_{\mathrm{sp}}$, when the fluctuation energy can be ignored, we have

$\langle E\rangle_{t} \sim L_{\text {zero }} \sigma$,

essentially the energy required to produce a classical vortex tangle of length $L_{\text {zero }}$ (up to $O(1)$ factors from the logarithmic tails). Eq. (18) completes condition (II) in order to relate line-zeroes with separation length between defects. Although these line zeroes have the topological charge and the energy of classical vortices, they are not yet fully-fledged defects. However, by the end of the linear regime, when decoherence on the scale $\xi_{\text {zero }}(t)$ has been effected, the final coupling of radial to angular modes that turns these protovortices into vortices incurs no significant energy change, and $n_{\text {zero }}$ of Eq. (15) is a reliable guide for the initial vortex density. We stress that, as far as counting vortices is concerned, all that matters is how the power in the field fluctuations is distributed. The distance between defects is the relevant wavelength, and not the defect size, which shows no decoherence.

This distance can be computed from the two point function of the field (see Eq. (15)). Up to logarithms in $\tau_{\mathrm{Q}}$ and the other parameters of the theory it is given by $[5,14,18]$,

$\xi_{\text {zero }}\left(t \approx t_{\text {sp }}\right)=\bar{\xi}_{\text {zero }} \approx \mu^{-1}\left(\tau_{\mathrm{Q}} \mu\right)^{1 / 3}$,

in accordance with the predictions of Kibble [4], but for very different reasons. Kibble correctly argues that the long-distance adiabatic correlation length $\xi_{\text {eq }}$ (that would diverge at $T=T_{\mathrm{c}}$ ) freezes in at time $t_{\mathrm{K}}$ before the transition with value $\bar{\xi}$, and this sets the scale for defect separation. However, in our picture defects do not form until time $t_{\mathrm{sp}}$ after the transition with a separation given by that of line-zeroes, that is arbitrarily small at time $t_{\mathrm{K}}$, and at the transition itself. That this is directly related to $\bar{\xi}$ is a consequence of dimensional analysis. This constraint was used to bound the production of monopoles [21] and cosmic strings [22] (vortices) in the early universe. (If we had broken an $O(3)$ symmetry $(a=1,2,3)$ the corresponding defects would be global monopoles, giving qualitatively similar conclusions.) This is all for flat space-time.

In summary, the mechanism for the production of classical vortices that we have proposed here has several parts. Firstly, the environment renders the long- wavelength modes, wavenumber $k_{0}$, of the orderparameter field classical at early times $t_{\mathrm{D}}\left(k_{0}\right)$, by or before the transition is complete at time $t_{\mathrm{sp}}$. In particular, those on the scale of the separation of the linezeroes that will characterise the classical defects will have decohered, even though the field modes on the scale of classical vortex thickness do not decohere. For all that, the field possesses classical correlations at early times by virtue of the quasi-Gaussian nature of the regime. For the longer-wavelength modes the classical behaviour of the field is expressed through the classical Langevin stochastic equations that it satisfies. However, for line-zeroes to mature into vortex cores classical stochastic equations (6) or, equivalently, classical Fokker-Planck equations are not enough. The field needs to have an energy profile commensurate with the vortex solutions to the ordinary classical Euler-Lagrange equations (3). This requires that the field fluctuations are peaked around long-wavelengths, to avoid fluctuations causing wiggles in the cores and creating small cortex loops, a related condition satisfied in our models. The resultant density of line-zeroes can already be inferred in the linear regime, whose topological charges are well-defined even though close inspection of their interior structure is not permitted classically.

The final result for their density, at the time $t_{\mathrm{sp}}$ of their formation is, up to logarithms, that proposed by Kibble [4] from dimensional analysis of meanfield theory, although the reasoning is very different. Although logarithms introduce different scales in principle, in practice they have no qualitative effect. It is apparent that vortices are not created from thermal fluctuations in the Ginzburg regime, as suggested in the first instance by Kibble in an earlier paper [1].

\section{Acknowledgements}

F.C.L. and F.D.M. were supported by Universidad de Buenos Aires, CONICET (Argentina), Fundación Antorchas and ANPCyT. R.J.R. would like to thank the European Science Foundation for support through its COSLAB programme, and the Rockefeller Foundation at Bellagio for hospitality, where this work was completed. 


\section{References}

[1] T.W.B. Kibble, J. Phys. A 9 (1976) 1387.

[2] W.H. Zurek, Phys. Rep. 276 (1996) 177.

[3] F.C. Lombardo, F.D. Mazzitelli, R.J. Rivers, hep-ph/0202041, to be published in: E. Verdaguer (Ed.), Proceedings of the 6th Conference on Quantum and Stochastic Gravity, String Cosmology and Inflation, Peyresq (2001), Int. J. Theor. Phys. (2002).

[4] T.W.B. Kibble, Phys. Rep. 67 (1980) 183.

[5] R.J. Rivers, J. Low Temp. Phys. 124 (2001) 41.

[6] A. Rajantie, Int. J. Mod. Phys. A 17 (2002) 1

[7] F.C. Lombardo, F.D. Mazzitelli, R.J. Rivers, Phys. Lett. B 523 (2001) 317.

[8] F.C. Lombardo, F.D. Mazzitelli, R.J. Rivers, hep-ph/0202042, to be published in: E. Verdaguer (Ed.), Proceedings of the 6th Conference on Quantum and Stochastic Gravity, String Cosmology and Inflation, Peyresq (2001), Int. J. Theor. Phys. (2002).

[9] F.C. Lombardo, F.D. Mazzitelli, R.J. Rivers, hep-ph/0204190, submitted to Phys. Rev. D (2002).

[10] F.C. Lombardo, F.D. Mazzitelli, Phys. Rev. D 53 (1996) 2001.

[11] M. Gleiser, R.O. Ramos, Phys. Rev. D 50 (1994) 2441.

[12] C. Greiner, B. Muller, Phys. Rev. D 55 (1997) 1026.

[13] D. Boyanovsky, H.J. de Vega, R. Holman, Phys. Rev. D 49 (1994) 2769;
D. Boyanovsky, H.J. de Vega, R. Holman, D.-S. Lee, A. Singh, Phys. Rev. D 51 (1995) 4419;

D. Boyanovsky, D. Cormier, H.J. de Vega, R. Holman, S. Prem Kumar, Phys. Rev. D 57 (1998) 2166.

[14] G. Karra, R.J. Rivers, Phys. Lett. B 414 (1997) 28.

[15] A.J. Gill, R.J. Rivers, Phys. Rev. D 51 (1995) 6949.

[16] M.J. Bowick, A. Momen, Phys. Rev. D 58 (1998) 085014.

[17] F.C. Lombardo, F.D. Mazzitelli, D. Monteoliva, Phys. Rev. D 62 (2000) 045016.

[18] E. Kavoussanaki, R.J. Rivers, G. Karra, Cond. Matt. Phys. 3 (2000) 133.

[19] B.I. Halperin, in: R. Balian, M. Kléman, J.-P. Poirier (Eds.), Physics of Defects, Proceedings of Les Houches, Session XXXV, 1980, NATO ASI Series, North-Holland, Amsterdam, 1981, p. 816.

[20] F. Liu, G.F. Mazenko, Phys. Rev. B 46 (1992) 5963.

[21] T.W.B. Kibble, Talk presented at the Meeting on Monopoles in Quantum Field Theory at ICTP, Trieste, December 1991 (Imperial College preprint); T.W.B. Kibble, E.J. Weinberg, Phys. Rev. D 81 (1991) 3188.

[22] R.H. Brandenberger, J. Maguiejo, astro-ph/0002030; R.H. Brandenberger, J. Maguiejo, in: Lecture Notes of the International School on Cosmology, Kish Island, Iran, January 22 - February 4, 1999, Large Scale Structure Formation, Kluwer, Dordrecht, 2000. 